\title{
The Relationship of Smoking-Opioid-Antidepressant Use and History of Spine Surgery
}

\author{
Walid Mohammad, Zaytseva Nadezhda \\ Medical Center of Central Georgia, Georgia, USA. \\ Email: mswalid@yahoo.com, zacev@yandex.ru \\ Received August $9^{\text {th }}, 2010$; revised August 11 $1^{\text {th }}, 2010$; accepted August $12^{\text {th }}, 2010$.
}

\begin{abstract}
Introduction: In a recent paper, smoking was shown to be an independent predictor of long-term continued opioid use in spine patients (Krebs et al. 2010). In this paper, we study the interaction of smoking with opioid and antidepressant use and the relationship with prior spine surgery and future spine surgery. Methods: Data on smoking, opioid and antidepressant use were retrospectively collected from 758 spine surgery inpatients [lumbar microdiskectomy (LMD), anterior cervical decompression and fusion $(A C D F)$ and lumbar decompression and fusion $(L D F)]$. Interaction with history of previous spine surgery and return for additional spine surgery was studied. Follow-up ranged from one to four years. Results: Significant correlation was detected between smoking and opioid use and between opioid and antidepressant use. History of previous spine surgery correlated with antidepressant use and return for additional spine surgery. Additional spine surgery correlated with opioid use and history of previous spine surgery. Smoking LMD patients on both opioids and antidepressants and smoking ACDF patients on antidepressants who have a history of prior spine surgery were more likely to return for additional spine surgery. However, in the LDF group, smoking patients on antidepressants without previous spine surgery were more likely to come for another spine surgery. Conclusion: Smoking-antidepressant-opioid use could be predictive of return for additional spine surgery during the follow-up years especially if a history of prior spine surgery was reported. Further research is needed on the implications of such an association.
\end{abstract}

Keywords: Spine Surgery, Smoking, Opioid, Antidepressant, Prior Spine Surgery, Additional Spine Surgery

\section{Introduction}

Chronic pain is a widespread health problem in the United States. Back pain patients are often uneasy patients who require multidisciplinary treatment and may undergo spine surgery, not once but several times. They usually need strong prescription painkillers and many of them resume opioid use for pain management after surgical intervention. A large percentage of back pain patients subsequently develop depression or carry depression as a comorbid diagnosis from the beginning. In a recent paper, smoking was found to be an independent predictor of long-term continued opioid use in lumbar disk hernia and spinal stenosis patients (1). Pursuant to that, we studied the interaction of smoking with opioid and antidepressant use and the relationship with prior spine surgery and future spine surgery.

\section{Materials and Methods}

The charts of 758 spine surgery patients operated betw- een Oct 2005 and Sep 2008 were retrospectively reviewed. The cohort included lumbar microdiskectomy (LMD), anterior decompression and fusion (ACDF) and lumbar decompression and fusion patients (LDF). Data on smoking, opioid and antidepressant use were collected (Tables 1 and 2). Prior and additional spine surgery were collected as categorical variables (Yes/No). Some patients may have had more than one spine surgery in the past or during follow-up (Figure 1). The relationship with history of previous spine surgery and return for additional spine surgery was studied using Spearman correlation. Follow-up was conducted for 1-4 years after the pivot surgery. Chi-square test was applied to compare percentages using SPSS v16.

\section{Results}

Significant correlations $(\mathrm{P}<0.05)$ were detected between smoking and opioid use and between opioid and antidepressant use (Table 3). History of previous spine surgery correlated with antidepressant use and return for addition- 
Table 1. Patient cohort per type of surgery, smoking and opioid-antidepressant use

\begin{tabular}{cccc}
\hline & & Frequency & Percent \\
\hline \multirow{3}{*}{ Type of surgery } & LMD & 234 & $\% 30.9$ \\
& ACDF & 305 & $\% 40.2$ \\
& LDF & 219 & $\% 28.9$ \\
& Total & 758 & $\% 100$ \\
Smoking & No & 517 & $\% 68.2$ \\
(Yes/No) & Yes & 241 & $\% 31.8$ \\
& Total & 758 & $\% 100$ \\
& None & 300 & $\% 39.6$ \\
Opioid-Antidepressant Use & Opioid & 249 & $\% 32.8$ \\
(Yes/NO) & Antidepressant & 96 & $\% 12.7$ \\
& Both & 113 & $\% 14.9$ \\
\hline
\end{tabular}

Table 2. Crosstabulation per type of surgery, smoking and opioid-antidepressant use

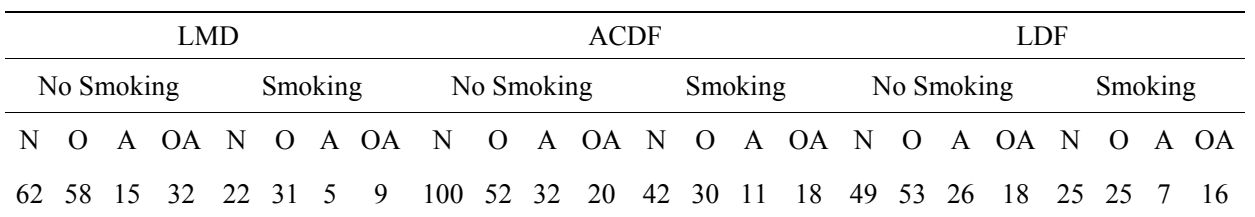

N: Neither Opioid Nor Antidepressant; O: Opioid; A: Antidepressant; OA: Opioid \& Antidepressant.
Prior Spine Surgery
Prior Spine Surgery
Additional Spine Surgery (Yes/No)
Data Collection
(Yes/No)

Figure 1. Schema explaining the structure of the study

Table 3. Spearman correlations

\begin{tabular}{|c|c|c|c|c|c|c|}
\hline & & Smoking & Antidepressant & Opioid & Prior Surgery & Additional Surgery \\
\hline \multirow[t]{3}{*}{ Smoking } & Coefficient & 1 & -0.003 & $0.079^{*}$ & 0.054 & 0.02 \\
\hline & Sig. (2-tailed) & . & 0.938 & 0.03 & 0.134 & 0.577 \\
\hline & $\mathrm{N}$ & 758 & 758 & 758 & 758 & 757 \\
\hline \multirow[t]{3}{*}{ Antidepressant } & Coefficient & -0.003 & 1 & $0.086^{*}$ & $0.098^{* *}$ & 0.018 \\
\hline & Sig. (2-tailed) & 0.938 & . & 0.014 & 0.005 & 0.608 \\
\hline & $\mathrm{N}$ & 758 & 816 & 816 & 816 & 815 \\
\hline \multirow[t]{3}{*}{ Opioid } & Coefficient & $0.079^{*}$ & $0.086^{*}$ & 1 & 0.06 & $0.079^{*}$ \\
\hline & Sig. (2-tailed) & 0.03 & 0.014 & . & 0.087 & 0.025 \\
\hline & $\mathrm{N}$ & 758 & 816 & 816 & 816 & 815 \\
\hline \multirow[t]{3}{*}{ Prior Surgery } & Coefficient & 0.054 & $0.098^{* *}$ & 0.06 & 1 & $0.142^{* *}$ \\
\hline & Sig. (2-tailed) & 0.134 & 0.005 & 0.087 & . & 0 \\
\hline & $\mathrm{N}$ & 758 & 816 & 816 & 816 & 815 \\
\hline \multirow[t]{3}{*}{ Additional Surgery } & Coefficient & 0.02 & 0.018 & $0.079^{*}$ & $0.142^{* *}$ & 1 \\
\hline & Sig. (2-tailed) & 0.577 & 0.608 & 0.025 & 0 & . \\
\hline & $\mathrm{N}$ & 757 & 815 & 815 & 815 & 815 \\
\hline
\end{tabular}


al spine surgery $(\mathrm{P}<0.01)$. Additional spine surgery correlated with opioid use $(\mathrm{P}<0.05)$ and history of previous spine surgery $(\mathrm{P}<0.01)$.

Opioid use was more common in the smoking groups of all three types of surgery candidates (Figure 2) but an- tidepressant use was more common in the smoking groups of ACDF and LDF patients but not in LMD patients (Figure 3). Statistically, there were significant differences $(\mathrm{P}<0.05)$ in opioid use and in simultaneous opioid-antidepressant use between smoking and non-

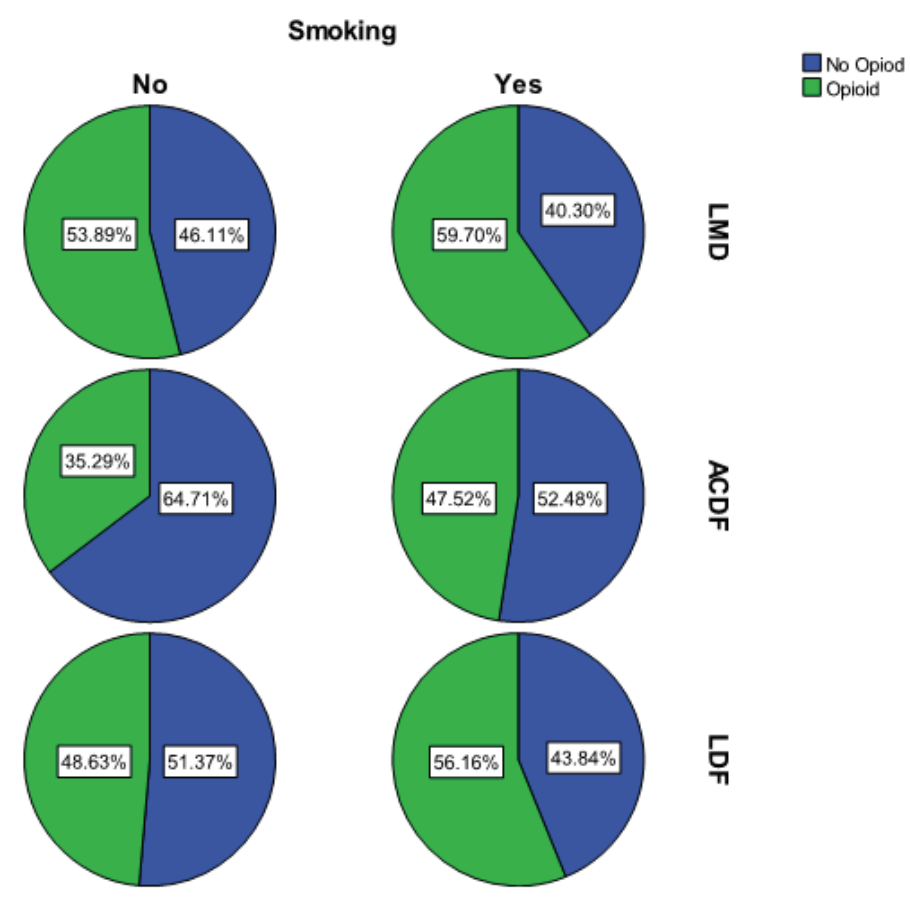

Figure 2. Prevalence of opioid use in three different types of spine surgery patients per smoking status

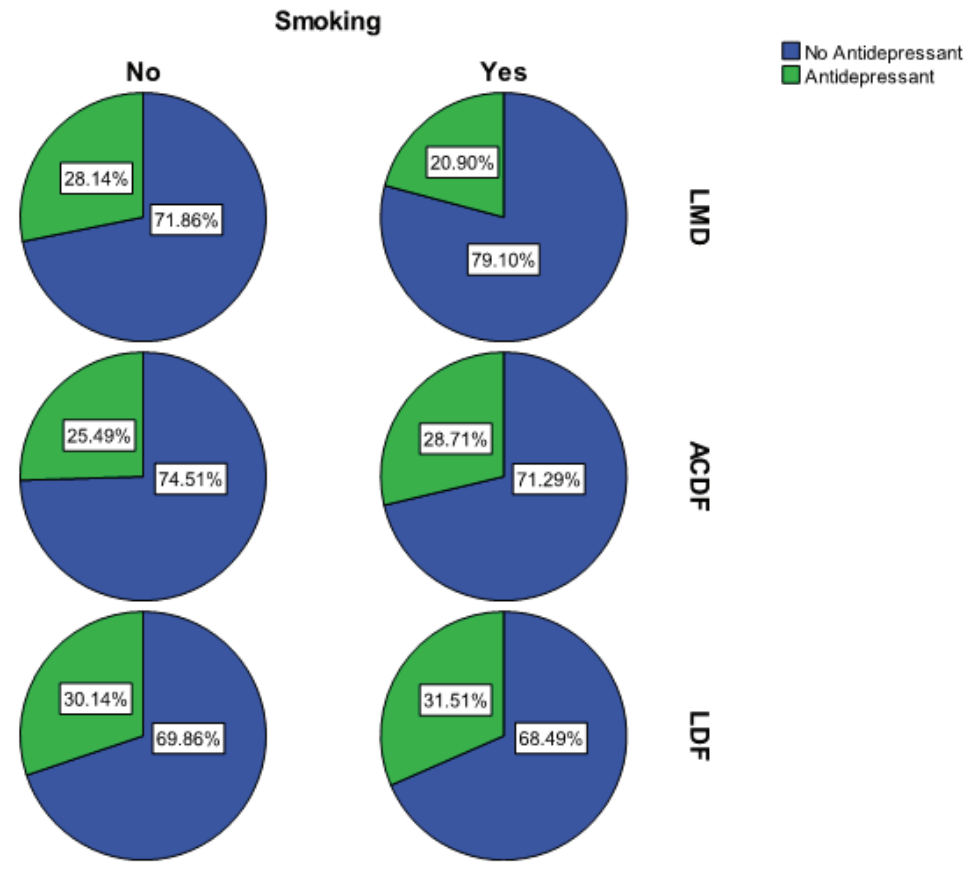

Figure 3. Prevalence of antidepressant use in three different types of spine surgery patients per smoking status 
smoking patients in the ACDF group only (Figure 4).

In the LMD group (Figure 5), smoking patients on both opioids and antidepressants with a history of previous spine surgery were more likely to return for addi-

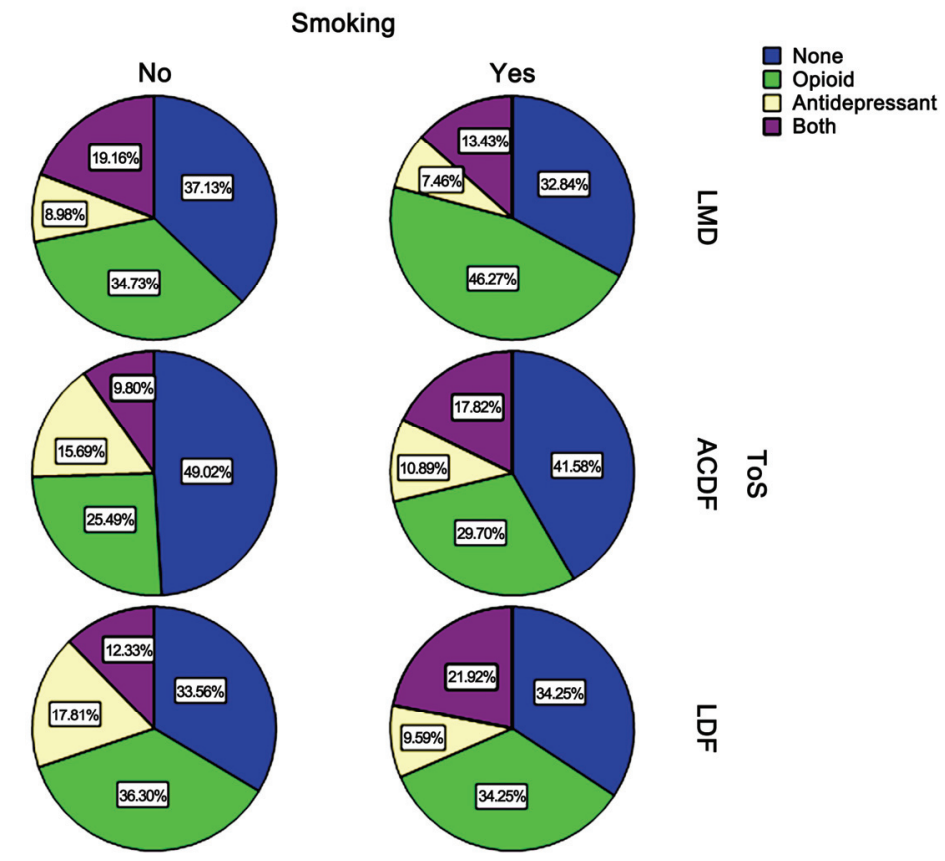

Figure 4. Prevalence of opioid-antidepressant use in three different types of spine surgery patients per smoking status

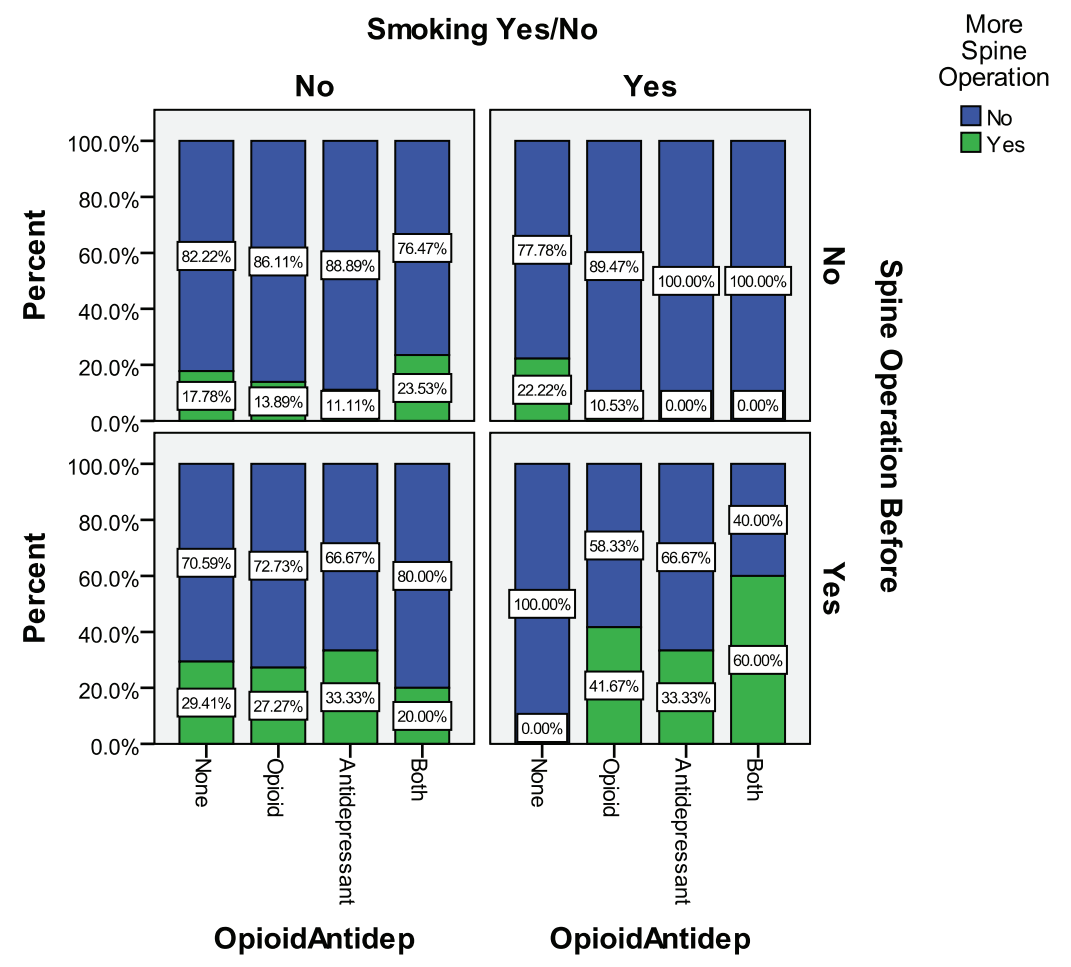

Figure 5. Prevalence of additional spine surgery in the LMD group per smoking, opioid-antidepressant use, and history of prior spine surgery 
tional spine surgery $(60.00 \%)$, followed by patients on opioids $(41.57 \%)$.

In the ACDF group (Figure 6), smoking patients on antidepressants with previous spine surgery were more likely to come for another spine surgery $(66.67 \%)$ followed by patients on opioids, whether smoking $(54.55 \%)$ or nonsmoking $(45.45 \%)$.

In the LDF group (Figure 7), smoking patients on an-

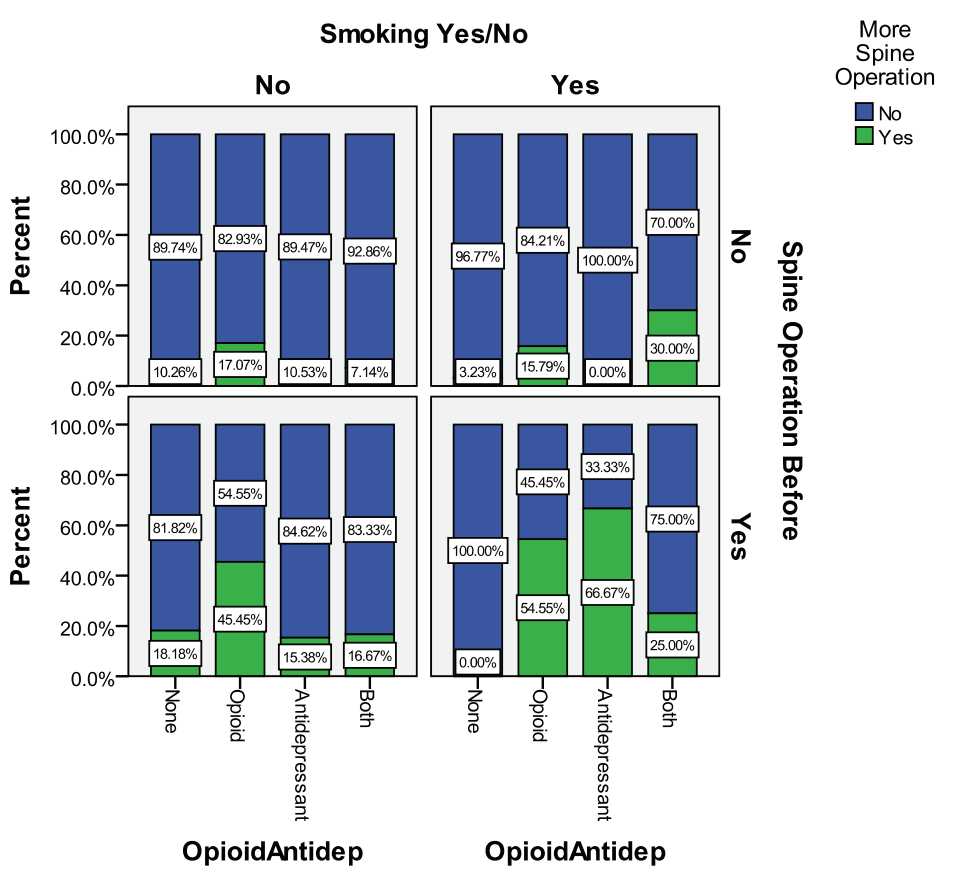

Figure 6. Prevalence of additional spine surgery in the ACDF group per smoking, opioid-antidepressant use, and history of prior spine surgery

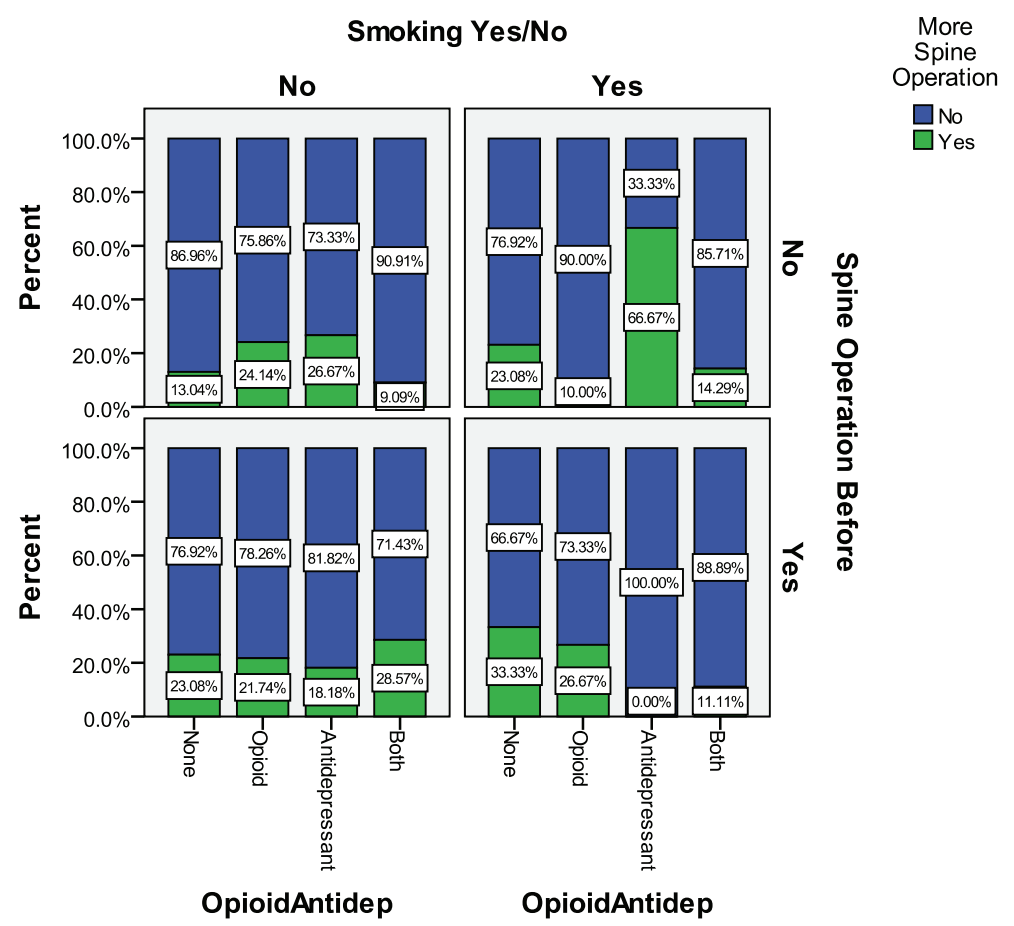

Figure 7. Prevalence of additional spine surgery in the LDF group per smoking, opioid-antidepressant use, and history of prior spine surgery 
tidepressants without previous spine surgery were more likely to return for another spine surgery (66.67\%).

\section{Discussion}

Smoking is a common "social" habit with known addictive inclinations that has clinical implications in different organ systems. In spine surgery, it has been associated with a negative effect on fusion rates [1]. We studied the interaction of this habitual use with other possibly-addictive psychotropic substances that chronic back pain patients are frequently prescribed. Opioids are known to be commonly used by back pain patients especially in the Western World [2]. The literature reports that patients with lumbar disc herniation or spinal stenosis use opioids in $42 \%$ of cases at baseline, $25 \%$ at 12 months, and $21 \%$ at 24 months [3]. Likewise, antidepressants are being increasingly utilized in the management of chronic pain patients [4]. Based on our results, there seems to be a correlation between smoking and opioid-antidepressant use in chronic back pain patients. History of previous spine surgery in a smoking patient with antidepressant or opioid use can predict future return for additional spine surgery.

Our study sheds light on an important aspect of the wide trend of chronic back pain. Knowing that antidepressants are the most commonly prescribed medications in the United States [5], many of these chronic patients may have depression or secondary gain issues inherent in their spine complaints. Smoking with chronic opioid-antidepressant use may thus reflect a deep psychosomatic disorder that can play a central role in the so-called "fai- led back syndrome". We recommend routine involvement of psychologists and psychiatrists with chronic previously-operated back pain patients who return for additional spine surgery in order to increase treatment efficiency and decrease the risk of failed surgery.

\section{REFERENCES}

[1] T. Andersen, F. B. Christensen, B. L. Langdahl, C. Ernst, S. Fruensgaard, J. Ostergaard, J. L. Andersen, S. Rasmussen, B. Niedermann, K. Høy, P. Helmig, R. Holm, B. E. Lindblad, E. S. Hansen, N. Egund and C. Bünger, "Fusion Mass Bone Quality after Uninstrumented Spinal Fusion in Older Patients," European Spine Journal, 2010, in Press.

[2] A. D. Furlan, J. A. Sandoval, A. Mailis-Gagnon and E. Tunks, "Opioids for chronic noncancer pain: A MetaAnalysis of Effectiveness and Side Effects," Canadian Medical Association Journal, Vol. 174, No. 11, 2006, pp. 1589-1594.

[3] E. E. Krebs, J. D. Lurie, G. Fanciullo, T. D. Tosteson, E. A. Blood, T. S. Carey and J. N. Weinstein, "Predictors of Long-Term Opioid Use among Patients with Painful Lumbar Spine Conditions," Journal of Pain, Vol. 11, No. 1, 2010, pp. 44-52.

[4] H. N. Chan, J. Fam and B. Y. Ng, "Use of Antidepressants in the Treatment of Chronic Pain," The Annals, Academy of Medicine, Singapore, Vol. 38, No. 11, 2009, pp. 974-979.

[5] E. Cohen, "CDC: Antidepressants most Prescribed Drugs in U.S," CNN Health, 2007. http://www.cnn.com/2007/ HEALTH/07/09/antidepressants/ 